\title{
Magnetostriction-driven cantilevers for dynamic atomic force microscopy
}

\author{
M. Penedo, I. Fernández-Martínez, ${ }^{\text {a) }}$ J. L. Costa-Krämer, M. Luna, and F. Briones \\ Instituto de Microelectrónica de Madrid (CNM, CSIC), Isaac Newton 8, 28760 Tres Cantos, \\ Madrid 28760, Spain
}

(Received 29 July 2009; accepted 30 August 2009; published online 9 October 2009)

\begin{abstract}
An actuation mode is presented to drive the mechanical oscillation of cantilevers for dynamic atomic force microscopy. The method is based on direct mechanical excitation of the cantilevers coated with amorphous $\mathrm{Fe}-\mathrm{B}-\mathrm{N}$ thin films, by means of the film magnetostriction, i.e., the dimensional change in the film when magnetized. These amorphous magnetostrictive $\mathrm{Fe}-\mathrm{B}-\mathrm{N}$ thin films exhibit soft magnetic properties, excellent corrosion resistance in liquid environments, nearly zero accumulated stress when properly deposited, and good chemical stability. We present low noise and high resolution topographic images acquired in liquid environment to demonstrate the method capability. (c) 2009 American Institute of Physics. [doi:10.1063/1.3237180]
\end{abstract}

Dynamic atomic force microscopy (DAFM) has proven to be a powerful technique for imaging soft biological samples in their native environment. ${ }^{1,2}$ The key element for the success of this operation method is the mechanical oscillation of the cantilever. In liquids, AFM cantilevers are commonly driven by either mechanical (with a piezoelectric ceramic) or magnetic (applying an alternating magnetic field gradient to a magnetized cantilever) excitation methods. When using piezoelectric excitation, the liquid acts as a coupling medium between the source of acoustic excitation and the liquid cell assembly. This effect results in amplitude versus frequency resonance curves usually described as a "forest of peaks," 3 in which the existence of various resonances makes the determination of the true cantilever resonances problematic. By using magnetic excitation, the cantilever is directly driven, avoiding unwanted mechanical coupling with the liquid cell and also avoiding the remnant oscillations of the cantilever base and glass support. The magnetic drive of AFM cantilevers in liquids has been extensively used in the last years either by coating the cantilevers with a thin ferromagnetic layer ${ }^{4}$ or by gluing a small magnetic particle at the end of the cantilever. ${ }^{5}$ The main drawback of the magnetic drive is that ferromagnetic material oxidizes in physiological environments, limiting the life time of the cantilevers.

In this work we demonstrate a method to drive the mechanical oscillation of the AFM cantilevers based on the magnetostrictive effect. Magnetostriction is the dimensional change in a magnetic material resulting from a change in its magnetic state. This is a well known effect, as magnetostrictive materials such as Terfenol-D (Ref. 6) or Galfenol (Ref. 7) have been used for applications such as actuators, sensors, or switches with success. Herein it is proved that the magnetostrictive effect on thin films can be applied to the mechanical actuation of AFM cantilevers. This method was proposed in a patent, ${ }^{8}$ although the magnetostrictive material used in this work is different from the ones suggested within that patent and also the configuration of the electromagnets, since we apply the magnetic field in the plane of the cantilever. The cantilevers are coated on the surface opposed to the tip side with a magnetostrictive $\mathrm{Fe}-\mathrm{B}-\mathrm{N}$ amorphous thin film. In the presence of a magnetic field, the magnetostrictive film

${ }^{a)}$ Electronic mail: ivan@imm.cnm.csic.es. expands forcing the cantilever to bend. An alternating magnetic field produces cyclic expansions of the magnetostrictive layer inducing the oscillatory movement of the cantilever. Amorphous $\mathrm{Fe}-\mathrm{B}-\mathrm{N}$ meets a combination of favorable properties: a good magnetostrictive effect is produced at very low applied magnetic fields $(<50 \mathrm{Oe})$, together with excellent mechanical and noncorrosive properties, which grant the cantilevers with long time stability. Fe-B-N amorphous thin films were deposited in a triode sputtering system from a nominally $\mathrm{Fe}_{80} \mathrm{~B}_{20}$ commercial target in the presence of an $\mathrm{Ar}+\mathrm{N}_{2}$ atmosphere. By in situ stress measurement, ${ }^{9}$ we have determined the optimal deposition conditions to achieve films with nearly zero accumulated stress. A target potential of $-0.75 \mathrm{kV}$, an $\mathrm{Ar}+\mathrm{N}_{2}$ pressure of $8 \cdot 10^{-3} \mathrm{mbar}$, and a $\mathrm{Fe}-\mathrm{B}-\mathrm{N}$ thin film nitrogen concentration of $7 \%$, as measured by non-Rutherford backscattering spectroscopy experiments (non-RBS). ${ }^{10}$

Under these deposition conditions, commercial cantilevers were coated with thicknesses up to $50 \mathrm{~nm}$. The coated cantilevers, whose force constants ranged from 0.08 to 3 $\mathrm{N} / \mathrm{m}$, show a maximum curvature lower than 1\%. These sputtered $\mathrm{Fe}-\mathrm{B}-\mathrm{N}$ thin films present a saturation magnetostriction coefficient of $\lambda_{S}=\delta l /\left.l\right|_{S}=+\left(35 \times 10^{-6}\right)$, combined with soft magnetic properties. ${ }^{10}$ In addition, the incorporation of nitrogen during deposition enhances the corrosion resistance in water, physiological buffer, and most solvents. ${ }^{11}$ A scheme of the magnetostrictive drive for DAFM is shown in Fig. 1. The experimental setup developed consists of a commercial AFM (Nanotec Electronica S.L.) ${ }^{12}$ with a specially designed liquid cell, in which a pair of electromagnets are placed to provide a magnetic field in a plane parallel to

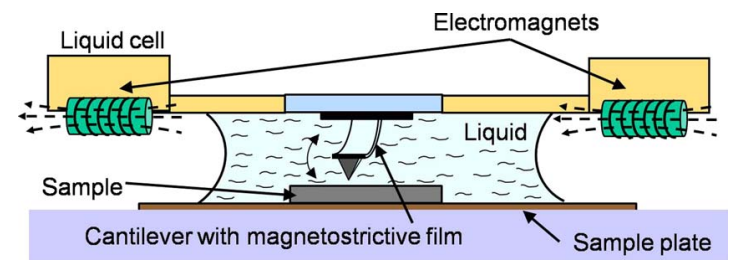

FIG. 1. (Color online) Scheme of the dynamic AFM driven by magnetostriction. The electromagnets produce an alternating magnetic field in a nearly parallel plane to the one defined by the cantilever (parallel to the plane defined by the sample) and in the direction of the short side of the rectangular cantilever. 


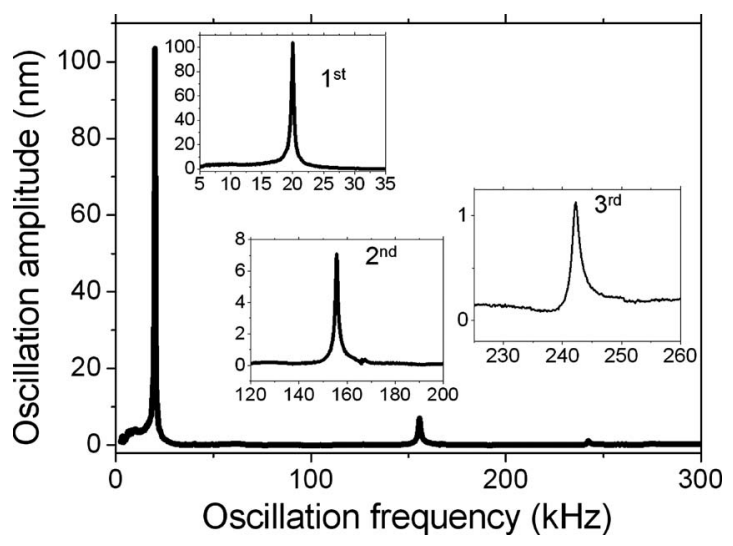

FIG. 2. Oscillation amplitude spectra taken in air of a silicon cantilever driven by the magnetostrictive actuation technique $(50 \mathrm{~mA}$ of current in the electromagnets, $50 \mathrm{~nm}$ of magnetostrictive coating). It is important to note that the frequency represented in the $x$ axis of the graphs corresponds to twice the excitation frequency of the applied magnetic field. The insets show a zoom in for the first three flexural modes $(20.01,155.70$, and $242.25 \mathrm{kHz})$.

the sample. Due to the geometrical design constraints in most liquid cells, it is more convenient to apply the magnetic field in a direction parallel to the direction defined by the short side of the rectangular cantilever. Therefore, during the sputtering deposition process, the direction of the cantilever short side is chosen as the magnetization hard axis of the magnetic coating (i.e., the axis direction at which the magnetization reverse by domains rotation) to maximize the magnetostrictive strain. ${ }^{13}$

An alternating current with an intensity that ranges between a few $\mathrm{mA}_{p-p}$ up to $50 \mathrm{~mA}_{p-p}$ drives the electromagnets and generates an alternating magnetic field of $25 \mathrm{Oe}$ at $50 \mathrm{~mA}_{p-p}$, as measured by a Gaussmeter in the low frequency regime. The electromagnets are fixed to the AFM and so the intensity of the applied magnetic field is thus independent of the tip sample separation. As expected from a magnetostrictive strain, the coated cantilever oscillates at twice the frequency of the applied magnetic field at which it is exposed $\left(\omega_{\text {oscillation }}=2 \cdot \omega_{\text {field }}\right) \cdot{ }^{14}$ This is due to the fact that the magnetostrictive effect is an even phenomenon with respect to the magnetic field, i.e., the magnetostrictive material expands independently of the sign of the magnetic field applied. The frequency spectrum of the cantilever oscillation is

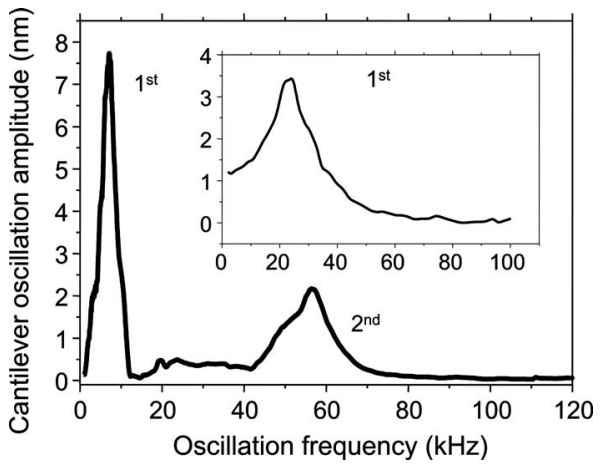

FIG. 3. Oscillation amplitude spectra of the cantilever immersed in water driven by the magnetostrictive effect (same cantilever and current as shown in Fig. 2). The main resonance frequency has dropped from $20.01 \mathrm{kHz}$ in air to $7.10 \mathrm{kHz}$ when the cantilever is immersed in water. The inset shows the oscillation amplitude spectra in water of a silicon nitride cantilever $(25 \mathrm{~mA}$ of current in the electromagnets, $50 \mathrm{~nm}$ of magnetostrictive coating). The first resonance peak is at $21.60 \mathrm{kHz}$.

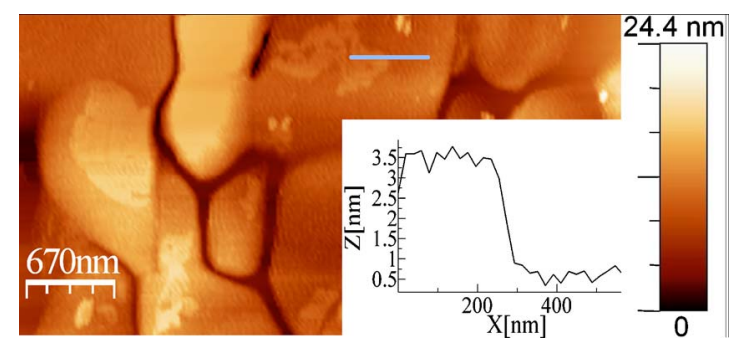

FIG. 4. (Color online) Dynamic atomic force microscopy topographic image of thiol islands $\left(\mathrm{C}_{22}\right)$ adsorbed on ultra flat gold (111) grains, obtained with magnetostrictive drive with the sample immersed in water. The islands measure approximately $3 \mathrm{~nm}$ in height, as shown in the profile.

shown in Fig. 2 in air and in Fig. 3 in liquid environment. The cantilever oscillation amplitude is represented as a function of the cantilever mechanical oscillation frequency $\omega_{\text {oscillation }}$, where $\omega_{\text {oscillation }}=2 \cdot \omega_{\text {field }}$.

In Fig. 2, the first three flexural oscillation modes of the silicon cantilever ${ }^{15}$ are clearly observed. As expected, the oscillation amplitude at resonance is damped when the cantilever is immersed in liquid (in Fig. 3 the quality factor of the first mode is reduced from 130 to 4), while the resonance frequencies shift to lower values. In the inset of Fig. 3 the oscillation amplitude spectra of a silicon nitride cantilever ${ }^{16}$ immersed in water is also shown. The curves of Fig. 3 demonstrate that the spectra obtained from magnetostrictive actuation method are free from the spurious liquid cell resonances that appear in the acoustic drive method, and cantilever resonant peaks are clearly observed despite the decrease in $Q$ in liquid environment.

It was also experimentally observed both in air and in liquid that the cantilever oscillates also at the frequency of the applied magnetic field $\left(\omega_{\text {oscillation }}=\omega_{\text {field }}\right)$. This result can be explained by considering that the amplitude of the applied magnetic field is not enough to reverse the film magnetization.

We routinely analyze samples in DAFM by means of magnetostrictive actuation. We present two topographic images (Figs. 4 and 5) as examples of the low noise and high resolution performance of this magnetostrictive drive when the AFM is operated with the sample immersed in liquids. Figure 4 shows thiol islands $\left(\mathrm{C}_{22} \mathrm{SH}\right)$ self-assembled on $\mathrm{Au}$ (111). The islands measure approximately $3 \mathrm{~nm}$ in height, a

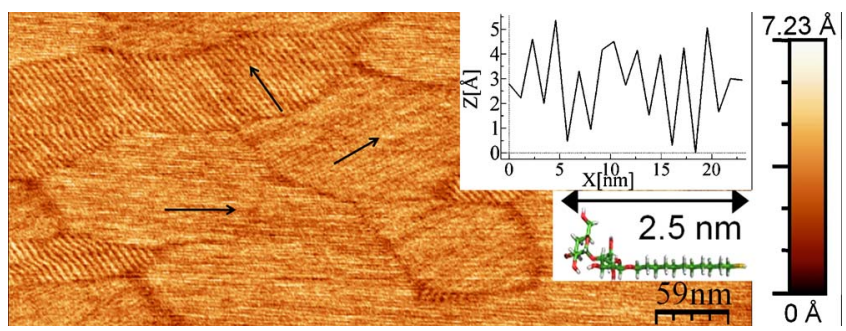

FIG. 5. (Color online) Dynamic atomic force microscopy topographic image of self-assembled maltoside neoglicoconjugate islands adsorbed on HOPG, obtained by means of magnetostrictive drive with the sample immersed in water. The organic molecules adsorb flat on graphite following the preferential crystallographic directions of the HOPG (hexagonal symmetry). The black arrows guide the eye in the identification of the different domain arrangements. The profile in the inset corresponds to the white line drawn in the image. A periodic pattern of $2.5 \mathrm{~nm}$ is observed $(17.23 \mathrm{~nm}$ between the two points shown/seven peaks), which corresponds to the length of the molecule. 
value which is close to the length of the thiols in solution ${ }^{17}$ (e.g., $2.8 \mathrm{~nm}$ ). Figure 4 shows a topographic image of selfassembled maltoside neoglicoconjugate [malto $\left.\mathrm{C}_{11} \mathrm{~S}\right]_{2}$ domains formed on Highly Ordered Pyrolytic Graphite (HOPG). The organic molecules adsorb flat on graphite under water following the preferential crystallographic directions of the HOPG. The profile (white line in the image) shows a periodic pattern of $2.5 \mathrm{~nm}$, which corresponds to the length of the molecule. ${ }^{18}$ The excellent lateral resolution achieved in this image indicates that the tip sharpness is maintained during the cantilever deposition process.

In summary, an original cantilever drive technique for DAFM is presented. Commercial cantilevers are coated with sputtered thin $\mathrm{Fe}-\mathrm{B}-\mathrm{N}$ films, which exhibit excellent magnetostrictive properties, good corrosion resistance when immersed in liquids, and nearly zero accumulated stress when properly deposited. This technique is especially advantageous in liquid environments, allowing the excitation of higher resonance modes and the dynamic operation of the AFM with low noise and high resolution.

We gratefully acknowledge Professor A. Raman for fruitful discussions, Professor C. Ocal and Professor C. Munuera for the preparation of the thiol islands sample, and Professor S. Penades for the synthesis of the maltoside neoglycoconjugate molecules. This work has been supported by the Ministry of Education and Science (MEC) of Spain through the Project Nos. CTQ2005-07993-C0202/BQU, NAN2004-09125-C07-02, and PET2007_0315. M.L. and M.P. acknowledge financial support from the MEC.
${ }^{1}$ S. Kasas, N. H. Thomson, B. L. Smith, H. G. Hansma, X. Zhu, M. Guthold, C. Bustamante, E. T. Kool, M. Kashlev, and P. K. Hansma, Biochemistry 36, 461 (1997).

${ }^{2}$ F. Moreno-Herrero, J. Colchero, J. Gómez-Herrero, and A. M. Baró, Phys. Rev. E 69, 031915 (2004).

${ }^{3}$ J. Kokavecz and Á. Mechler, J. Vac. Sci. Technol. A 11, 808 (1993).

${ }^{4}$ W. Han, S. M. Lindsay, and T. Jing, Appl. Phys. Lett. 69, 4111 (1996).

${ }^{5}$ S. P. Jarvis, T. P. Weihs, A. Oral, and J. B. Pethica, Mater. Res. Soc. Symp. Proc. 308, 127 (1993).

${ }^{6}$ A. E. Clark, Ferromagnetic Materials (North-Holland, Amsterdam, 1980), Vol. 1.

${ }^{7}$ A. E. Clark, J. B. Restoff, M. Wun-Fogle, T. A. Logasso, and D. L. Schlagel, IEEE Trans. Magn. 36, 3238 (2000).

${ }^{8}$ S. M. Lindsay and T. Jing, U.S. Patent No. 6,121,611 (19 September 2000).

${ }^{9}$ I. Fernández-Martínez, J. L. Costa-Krämer, and F. Briones, J. Appl. Phys. 103, 113902 (2008)

${ }^{10}$ I. Fernández-Martínez, M. S. Martín-González, R. González-Arrabal, R. Álvarez-Sánchez, F. Briones, and J. L. Costa-Krämer, J. Magn. Magn. Mater. 320, 68 (2008).

${ }^{11}$ H. Karamon, T. Masumoto, and Y. Makino, J. Appl. Phys. 57, 3527 (1985).

${ }^{12}$ Nanotec Electrónica S.L. www.nanotec.es.

${ }^{13}$ I. Fernández-Martínez, Ph.D. thesis, Universidad Autónoma de Madrid, 2008.

${ }^{14}$ B. D. Cullity and C. D. Graham, Introduction to Magnetic Materials, 2nd ed. (Wiley, New York, 2008)

${ }^{15}$ Cantilever purchased from Mikromasch, CSC38/AIBS, force constant $=0.08 \mathrm{~N} / \mathrm{m}$.

${ }^{16}$ Cantilever purchased from Olympus, ORC8-PS, force constant $=0.76 \mathrm{~N} / \mathrm{m}$

${ }^{17}$ S. Xu, P. E. Laibinis, and G. Liu, J. Am. Chem. Soc. 120, 9356 (1998).

${ }^{18}$ M. Fuss, M. Luna, D. Alcántara, J. M. de la Fuente, P. M. Enríquez-Navas, J. Angulo, S. Penades, and F. Briones, J. Phys. Chem. B 112, 11595 (2008). 